\title{
Correlación entre el diagnóstico clínico de un sangrado uterino anormal y el resultado anatomopatológico
}

\author{
Correlation between clinical diagnosis of abnormal uterine \\ bleeding and anatomopathological outcome
}

\author{
Daryl Haydeé Colín Mejía, ${ }^{*}$ Catalina Romo Aguirre
}

\section{Resumen}

Introducción: El sangrado uterino anormal es la segunda causa de consulta ginecológica, cuyo diagnóstico o tratamiento, muchas veces, puede estar relacionado con enfermedades oncológicas o enfermedades que requieren abordaje quirúrgico para su tratamiento, por lo que éste suele ser urgente, y de ahí que el diagnóstico clínico sea muy importante. Material y métodos: Estudio transversal, observacional y retrospectivo que evaluó 276 mujeres en edad entre 25-60 años atendidas por sangrado uterino anormal en el Hospital Ángeles Pedregal, entre enero del 2014 y junio del 2017. De cada paciente, se recuperó de su expediente clínico el diagnóstico de ingreso, el obtenido mediante estudios de gabinete y el anatomopatológico. Resultados: Se estudiaron 276 casos de mujeres con una edad promedio de 42.9 años, de las cuales $39.7 \%$ no ha presentado un embarazo. El $47.6 \%$ de las intervenciones quirúrgicas se realizaron por laparoscopia. De los 261 casos de leiomioma diagnosticados por muestra de tejido, $91.5 \%$ fue diagnosticado clínicamente; sin embargo, sólo $11.26 \%$ de los casos de adenomatosis fueron identificados clínicamente. Conclusión: Pese a los avances en los algoritmos diagnósticos y el empleo de técnicas de gabinete, el diagnóstico anatomopatológico en el sangrado uterino anormal es vital para un diagnóstico preciso.

Palabras clave: Hemorragia uterina, leiomioma, patología, útero, miomas, sangrado, metrorragia, sangrado disfuncional, tumor uterino.

\footnotetext{
* Residente de Ginecología y Obstetricia del Hospital Ángeles Pedregal, Facultad Mexicana de Medicina, Universidad la Salle.

* Departamento de Anatomía Patológica, Hospital Ángeles Pedregal.
}

Correspondencia:

Daryl Haydeé Colín Mejía

Correo electrónico: dra.darylcolin@gmail.com

Aceptado: 06-06-2019.

www.medigraphic.com/actamedica

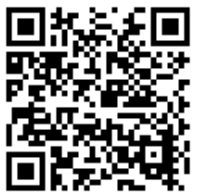

\section{Abstract}

Introduction: Abnormal uterine bleeding is the second cause of gynecological consultation and many times its diagnosis or treatment may be related to oncological diseases or require a surgical approach for treatment require immediate access so the clinical assessment is very important. Material and methods: Cross-sectional, observational and retrospective study that evaluated women between the ages of 25-60 years treated at the Ángeles Pedregal Hospital between January 2014 and June 2017 due to abnormal uterine bleeding. They required surgical intervention and a sample was sent for anatomopathological analysis as a diagnostic method. Results: We studied 276 cases of women of average age of 42.9 years, of which $39.7 \%$ have not presented a pregnancy. $56.23 \%$ of the deeds have been resolved by caesarean sections. $47.6 \%$ of the surgical interventions were performed by laparoscopy. Of the 261 cases of leiomyoma diagnosed by tissue sample, $91.5 \%$ were clinically diagnosed; however, only $11.26 \%$ of the cases of adenomatosis were clinically identified. Conclusion: Despite advances in diagnostic algorithms and the use of cabinet techniques, anatomopathological diagnosis in abnormal uterine bleeding is vital for an accurate diagnosis.

Keywords: Uterine hemorrhage, leiomyoma, pathology, uterus, myomas, bleeding, metrorrhagia, dysfunctional bleeding, uterine tumor.

\section{INTRODUCCIÓN}

El sangrado uterino anormal es una condición patológica frecuente, que tiene un impacto social y económico significativo, además de ser la segunda causa de consulta ginecológica después de las infecciones cervicovaginales. ${ }^{1}$ En México, del total de las mujeres que cursan con hemorragia uterina anualmente, sólo 6 millones de ellas buscan atención médica. El diagnóstico presuntivo se realiza mediante un interrogatorio y examen físico, para posteriormente confirmarse mediante estudios de 
laboratorio y gabinete (Guía de Práctica Clínica COMEGO, 2013).

Actualmente, algunos autores consideran que de 9 a $14 \%$ de las mujeres en edad reproductiva han tenido ciclos menstruales mayores a 80 mililitros de sangrado por ciclo menstrual. ${ }^{2,3}$ La hemorragia uterina en Estados Unidos representa la causa de cerca de 600,000 histerectomías anualmente. $^{3}$

La hemorragia uterina se define como el sangrado del cuerpo uterino anormal en volumen, regularidad, temporalidad (o los tres), que ha estado presente durante la mayor parte de los últimos seis meses, mientras que el sangrado intermensual se define como el que ocurre entre menstruaciones que son claramente definidas como cíclicas y predecibles; comprende la ocurrencia de episodios aleatorios, así como los que se manifiestan predeciblemente al mismo tiempo en cada ciclo y que pueden también ser considerados o no como hemorragia uterina. ${ }^{3}$ Sin embargo, debido a que su etiología es múltiple, su diagnóstico clínico puede verse limitado debido a la falta de un cuadro clínico específico para cada etiología, por lo cual el tratamiento inicial se establece de manera sintomática. Sin duda alguna, el diagnóstico clínico inicial es la base angular para determinar un tratamiento y abordaje específico, por lo que se debe buscar que exista una correlación adecuada entre el diagnóstico clínico con el diagnóstico del resultado del estudio de patología, con la finalidad de disminuir las cirugías innecesarias que conllevan a otras posibles complicaciones que podrían evitarse.

\section{MATERIAL Y MÉTODOS}

Se trata de un estudio transversal, observacional y descriptivo en el que se estudiaron 276 casos de mujeres

Figura 1: Frecuencia de número de gestaciones en los 276 casos estudiados.

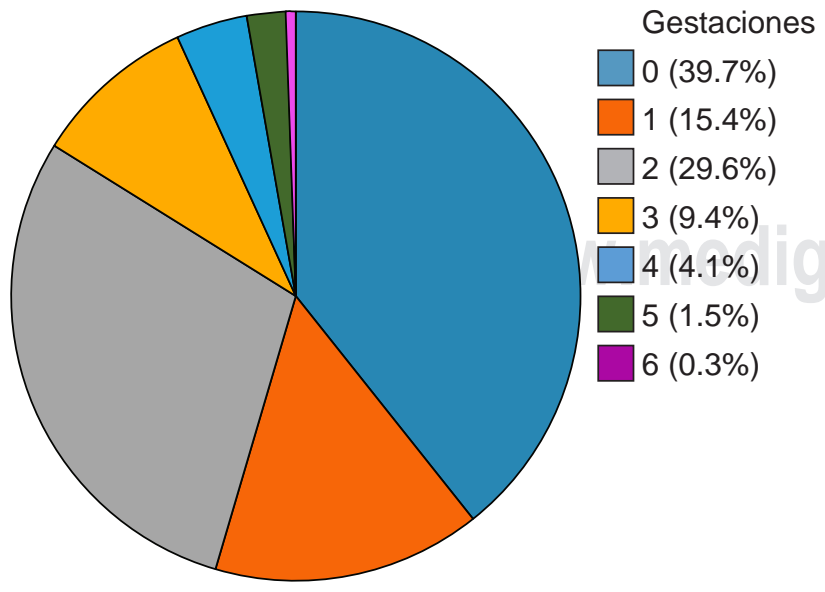

con sangrado uterino anormal acorde con la clasificación CIE-10 N938, cuya edad estuviese entre 25 y 60 años y que fuesen atendidas en el periodo comprendido del 1 de enero del 2014 al 30 de junio del 2017 en el Hospital Ángeles Pedregal.

De los 276 casos incluidos en el presente trabajo, se obtuvo de sus expedientes clínicos si fueron sometidos a algún procedimiento quirúrgico como parte del abordaje terapéutico; además que contaran con un diagnóstico postquirúrgico por el departamento de patología. Adicionalmente se revisó también el diagnóstico clínico de ingreso y el diagnóstico realizado mediante estudios de gabinete.

El análisis estadístico se realizó mediante el uso del programa IBM SPSS $\vee 25$ (USA).

\section{RESULTADOS}

La muestra se integró por 276 casos de mujeres con un rango de edad de 25-60 años, las cuales tuvieron una edad promedio de $42.9 \pm 7.07$ años. De éstas, 109 casos $(39.7 \%)$ correspondieron a mujeres nuligestas y $45 \%$ a multigestas (Figura 1).

Respecto a la presentación clínica de dolor pélvico crónico, éste sólo se presentó en 59.2\% (158 casos), sin embargo, sólo $51.26 \%$ de dichos casos recibió tratamiento analgésico previo a su atención.

Ahora bien, en relación con los diagnósticos realizados, se observó lo señalado en la siguiente tabla (Tabla 1). Con relación al diagnóstico clínico contra el realizado por patología, se observó que el $\mathbf{9 1 . 5 7 \%}$ de los casos de miomatosis uterina sí fue diagnosticado clínicamente; 100\% de los casos de quistes ováricos también fue identificado clínicamente, con un caso de falso positivo; mientras que $30.76 \%$ de los casos de pólipos endometriales fue identificado clínicamente. Sin embargo, por clínica sólo se diagnosticó $12.5 \%$ de los casos de endometriosis y $11.26 \%$ de los casos de adenomatosis. Interesantemente, respecto a la miomatosis uterina como diagnóstico al ingreso, se reportaron 226 casos, y de éstos se reportaron mediante gabinete 240, quirúrgicamente 253 diagnósticos y mediante análisis anatomopatológico se reportaron sólo 261 casos. Adicionalmente, no se encontró correlación significativa entre los antecedentes obstétricos y los resultados anatomopatológicos.

En cuanto al tipo de procedimientos, se realizaron (Figura 2) una histeroscopia $(0.4 \%), 127$ abordajes laparoscópicos $(47.6 \%)$, cinco abordajes de laparoscopia más histeroscopia (1.9\%) y 109 casos de laparotomía (40.8\%); 16 procedimientos fueron realizados mediante el uso de un robot laparoscópico (6\%) y ocho casos mediante cirugía vía vaginal (3\%). 
Tabla 1: Frecuencias de diagnósticos clínicos por gabinete, postquirúrgicos y anatomopatológicos.

$\begin{array}{lccrc} & \text { Diagnóstico clínico } & \begin{array}{c}\text { Diagnóstico por } \\ \text { gabinete }\end{array} & \begin{array}{c}\text { Diagnóstico } \\ \text { postoperatorio }\end{array} & \begin{array}{c}\text { Diagnóstico } \\ \text { anatomopatológico }\end{array} \\ \text { Adenocarcinoma } & 0 & 0 & 1 & 1 \\ \text { Adenomatosis } & 8 & 4 & 10 & 71 \\ \text { Endometriosis } & 1 & 1 & 5 & 8 \\ \text { Hidrosalpinge } & 0 & 1 & 1 & 1 \\ \text { Hiperplasia endometrial } & 0 & 11 & 9 & 6 \\ \text { Miomatosis uterina } & 239 & 240 & 253 & 261 \\ \text { Pólipo endometrial } & 8 & 11 & 8 & 26 \\ \text { Quiste ovárico } & 4 & 9 & 0 & 3 \\ \text { Sin diagnóstico } & 0 & 6 & 0 & 1 \\ \text { Tumor ovárico } & 1 & 1 & 8 & 0\end{array}$

\section{DISCUSIÓN}

La edad promedio en que se ha descrito la presencia de sangrado uterino anormal oscila entre 25 a 44 años, lo que resulta similar a lo encontrado en nuestro estudio. ${ }^{4}$ Esto se debe a que, a mayor edad el riesgo de sangrado aumenta considerablemente, lo cual está asociado con cambios hormonales. ${ }^{5}$

Como fue descrito en este estudio, cerca de $40 \%$ de los casos incluidos en la muestra era de mujeres nuligestas, lo cual es un factor de riesgo para el desarrollo de adenomatosis, cuyo diagnóstico está presente en un tercio de la población estudiada. ${ }^{6,7}$ Además, la presencia de adenomatosis también ha sido asociada con el desarrollo de tejido fibroso o miomatosis uterina, que fue el principal diagnóstico anatomopatológico en nuestro estudio. ${ }^{8}$

Respecto de la presencia de multigestas en nuestra población estudiada, se considera una situación epidemiológica común o frecuente en nuestro país, como se ha observado en Estados como Guadalajara o en los Estados del norte del país. ${ }^{9}$

La presentación clínica de dolor crónico como principal síntoma acompañante del sangrado uterino en cerca de la mitad de la población es un síntoma que a muchas mujeres las lleva a iniciar un tratamiento, a causa de la severidad del dolor y por interferir en sus vidas más que el propio sangrado; la principal solución al problema de sangrado uterino anormal es la histerectomía, para su posterior evaluación por anatomía patológica. ${ }^{10,11}$

Por último, se observa claramente que el diagnóstico clínico queda ínfimamente por debajo del diagnóstico anatomopatológico de adenocarcinomas, adenomatosis, endometriosis, hiperplasia endometrial y pólipo endometrial. Todos ellos son parte de la clasificación FIGO de sangrado uterino anormal, ${ }^{12}$ debido a su difícil diagnóstico clínico, ya que carece de un cuadro clínico especifico o único, por lo que el estudio anatomopatológico sigue siendo importante en el diagnóstico certero y preciso del sangrado uterino anormal.

\section{CONCLUSIÓN}

Pese a los avances en los algoritmos diagnósticos y el empleo de técnicas de gabinete, el diagnóstico anatomopatológico en el sangrado uterino anormal es vital para un diagnóstico preciso, además de que en el diagnóstico clínico y de gabinete suele considerarse a la miomatosis uterina como el único diagnóstico, aun cuando suele presentarse junto con otras alteraciones anatomopatológicas o en combinación con una gran gama de otros síntomas generales, los cuales son inespecíficos para poder llegar a un diagnóstico certero.

Figura 2: Procedimientos quirúrgicos realizados, agrupados por vía de abordaje.

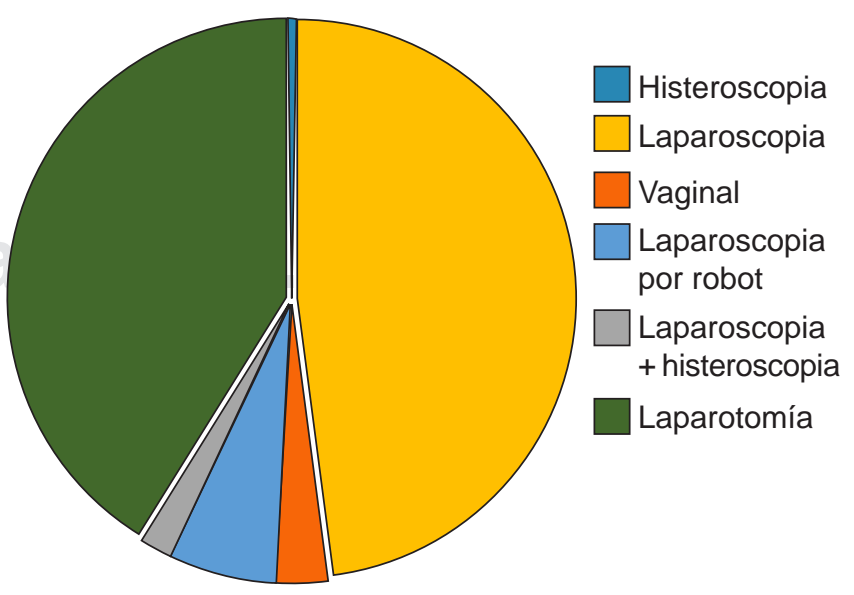




\section{REFERENCIAS}

1. Munro MG, Critchley HO, Fraser IS, Group FMDW. The FIGO classification of causes of abnormal uterine bleeding in the reproductive years. Fertil Steril. 2011; 95 (7): 2204-2208, 8 e1-3.

2. DeCherney AH Nathan L, Laufer N, Roman AS. Diagnóstico y tratamiento ginecoobstétricos .11a. ed. Mexico: McGraw Hill; 2014.

3. Munro MG, Critchley H, Fraser IJRdc. La clasificación FIGO de causas de sangrado uterino anormal en los años reproductivos. Revista del Climaterio. 2011; 15 (85): 9-17.

4. Palep-Singh M, Prentice A. Epidemiology of abnormal uterine bleeding. Best Pract Res Clin Obstet Gynaecol. 2007; 21 (6): 887890.

5. Moradan S, Ghorbani R, Lotfi A. Agreement of histopathological findings of uterine curettage and hysterectomy specimens in women with abnormal uterine bleeding. Saudi Med J. 2017; 38 (5): 497-502.

6. Vercellini P, Consonni D, Dridi D, Bracco B, Frattaruolo MP, Somigliana E. Uterine adenomyosis and in vitro fertilization outcome: a systematic review and meta-analysis. Hum Reprod. 2014; 29 (5): 964-977.
7. Abbott JA. Adenomyosis and Abnormal Uterine Bleeding (AUB-A)Pathogenesis, diagnosis, and management. Best Pract Res Clin Obstet Gynaecol. 2017; 40: 68-81.

8. Hulka CA, Hall DA, McCarthy K, Simeone J. Sonographic findings in patients with adenomyosis: can sonography assist in predicting extent of disease? AJR Am J Roentgenol. 2002; 179 (2): 379-383.

9. Panduro B, Guadalupe J, Pérez M, Jesús J, Panduro EG, Castro JF et al. Factores de riesgo prenatales en la muerte fetal tardía, Hospital Civil de Guadalajara, México. Rev Chil Obstet Gineco. 2011; 76 (3): 169-174.

10. Learman LA, Nakagawa S, Gregorich SE, Jackson RA, Jacoby A, Kuppermann M. Success of uterus-preserving treatments for abnormal uterine bleeding, chronic pelvic pain, and symptomatic fibroids: age and bridges to menopause. Am J Obstet Gynecol. 2011; 204 (3): 272 e1-7.

11. Carlson KJ, Miller BA, Fowler FJ, Jr. The Maine Women's Health Study: II. Outcomes of nonsurgical management of leiomyomas, abnormal bleeding, and chronic pelvic pain. Obstet Gynecol. 1994; 83 (4): 566-572.

12. Bacon JL. Abnormal Uterine Bleeding: Current Classification and Clinical Management. Obstet Gynecol Clin North Am. 2017; 44 (2): 179-193. 